\title{
Shared virtual presentation board for e-Meeting in higher education on the WebELS platform
}

\author{
Arjulie John Berena ${ }^{1 *}$, Sila Chunwijitra ${ }^{2}$, Hitoshi Okada ${ }^{1}$ and Haruki Ueno
}

\footnotetext{
* Correspondence: berena@nii.ac.jp ${ }^{1}$ National Institute of Informatics, Tokyo, Japan

Full list of author information is available at the end of the article
}

\begin{abstract}
In this paper, the development of a shared virtual presentation board (VPB) for real-time e-Meeting on the Web-based e-Learning System (WebELS) platform is introduced. WebELS is a general-purpose e-Learning system to support flexibility and globalization of higher education in science and technology. In WebELS, the Meeting module consists of online presentation and video conference system, and the combination of both allows the creation of a so-called virtual room for e-Meeting applications where participants convene via the Internet. Online presentation features synchronized slide control between the presenter and the listeners for slide changing, scrolling, zooming, cursor positioning, and playback control for video embedded on the slide. It also features online annotation that allows the presenter to write using a pen function on the slide display panel. The system has video conferencing function that provides an audio-video communication among the meeting participants. This paper discusses briefly the video conference system, and focuses on online presentation based on slide-based synchronization and the development of VPB. VPB is a shared object that resides on the server that is updated every time the presenter client makes mouse events and is periodically accessed by client system in order that listener's presentation viewer synchronized with that of the presenter.
\end{abstract}

Keywords: e-Learning, e-Meeting, Online presentation, Online annotation, Shared presentation board, WebELS

\section{Introduction}

Advancements of technology and the informatization of society are factors that paved the way for shifting the teaching methodologies in higher educational system from the traditional classroom-based method to the use of information and communications technology. In recent years, Internet-based teaching and learning technologies have been widely available enabling e-Learning to become a major form of educational methodology addressing time-limitation and location-limitation between teacher and students [1]. e-Learning infrastructure can be easily carried out because of the advancement in internetworking technologies, multimedia information processing technologies, and software technologies at lower cost and higher quality in a global scale [2].

Unlike e-Learning in middle school and undergraduate programs where course management and automated student assessment are typical features, in higher education, particularly in the graduate school, the system should support self-learning, group meetings, and research presentations [3-6]. The characteristics of an e-Learning system

(c) 2013 Berena et al.; licensee Springer. This is an Open Access article distributed under the terms of the Creative Commons Attribution License (http://creativecommons.org/licenses/by/2.0), which permits unrestricted use, distribution, and reproduction in any medium, provided the original work is properly cited. 
for higher education based on information technology perspective can be best deduced from the three areas as follows:

- Activities: A system must support online activities, such as group distance meetings, multi-point distribution of oral presentation in conferences, online collaboration with professors and other researchers in academe and industries, and other similar scenarios.

- Tools and Materials: A system must have powerful authoring tool so that professors and students alike can create and edit their own presentation materials on their personal computers. Users should be able to utilize various contents in the form of PDF, PPT and other office documents on various operating systems, such as Windows, Mac OS and Linux.

- Behavior: A system must support slide-based presentation and multimedia content, such as audio and video. Users should be able to make comment and annotation to the presentation material and must also be available during online meeting and discussion.

In carrying out the demands of higher education in view of information technology, an e-Meeting system is strongly requested rather than a typical learning management system (LMS) $[7,8]$. An effective e-Meeting system requires primary features like online slide presentation, online annotation, chat messaging and video conferencing system. Although there are a lot of similar systems, but it is very rare to find an all-in-one system that really answer the need to support e-Meeting for higher education.

In this paper, we discuss the development of a shared virtual presentation board (VPB) for e-Meeting system on the Web-based e-Learning System (WebELS) platform. VPB is a master copy of the presenter's presentation panel environment, stored onto the server and used to achieve slide presentation synchronization between the presenter and the listeners. Synchronization in our context refers to real-time mirroring of slide presentation events that include slide controls for slide changing, vertical and horizontal scrolling, panning, zooming, cursor positioning, annotation, and playback control for video embedded on the slide. The system has a video conferencing function that provides an audio-video communication among the meeting participants. An effective e-Meeting is made possible by combining the online slide presentation and video conference system to create a socalled virtual room, where meeting participants convene via the Internet.

\section{Review of online presentation technologies}

With the fast-paced development of Internet application technologies, various online presentation tools have become widely available. We describe some popular related technologies according to their core design, such as screen sharing, document sharing and slide synchronization. We also discuss the benefits and drawbacks for each in view of e-Meeting application for higher education.

\section{Screen sharing technology}

Screen sharing technology allows one to transmit the desktop static image of his computer to one or more remotely connected users in the network. Some screen sharing systems allow remote control which shares the ability to control the keyboard and mouse to other users. Some systems that use screen sharing are VNC [9], Skype [10], GoToMeeting [11], WebEx [12], TeamViewer [13], join.me [14], to name a few. 
The advantage of screen sharing technique is that presenter can show various applications on his desktop and be seen by other participants. In view of e-Meeting however, presenter have to be careful that sensitive information is not displayed as it can be visible to other meeting participants. Another drawback of screen-sharing technology is the reduced graphic quality that small text cannot be easily read or small objects cannot be easily recognized. Screen sharing technique works well in broadband, but may not operate well in narrowband as it utilizes greater bandwidth for transmitting of encoded pixel data of screen image. Bandwidth demand gets very high if there is a lot of pixel change at a short time, such as when scrolling a window or showing animated presentation may take several seconds to completely display the image at remote site. Another major drawback of screen sharing is that it requires advanced network address translation (NAT), firewall and router configuration such as port forwarding in order for the connection to go through. Other systems are also limited to peer-to-peer connection, and does not support multi-user. Since it is not a web-based system, it is necessary to download and install the software before screen sharing can start up. Some screen sharing systems have an integrated support to Voice over Internet Protocol (VoIP) or teleconferencing (telephone) to realize an e-Meeting. But others do not have and users have to use other VoIP system.

\section{Document sharing technology}

Document sharing tools are sometimes referred to as online presentation tool because it is possible to share presentation files online. Some document sharing tools allow one to create a PowerPoint presentation online or upload an existing PowerPoint file onto the server. Other users can then view and edit the shared presentation, such as Google Docs [15] and SlideRocket [16], while others allow anyone to view only but does not allow editing, such as authorSTREAM [17] and SlideShare [18]. Some systems support various file formats [15], but others only support PowerPoint [16-18]. Users sharing a document is not required to be online at a time, thus there is no real time interaction among the users. Document sharing does not employ integrated support for VoIP as the main purpose is to share documents.

\section{Slide-based synchronization technology}

Slide-based synchronization technology refers to mirroring of the presentation panel among the meeting participants, i.e., what the presenter see on his display panel will also be replicated on the listeners' display panel. Some popular systems that use slide synchronization are V-Cube [19], Adobe Connect [20], BigBlueButton [21], among others. During online presentation, MS PowerPoint or PDF file is uploaded on-the-fly by the client presenter to the server. At the server, the uploaded file is converted to an image format before it can be viewed by the users. For instance, however, the file document to be uploaded is considerably large, conversion process may take a lot of time, and this may cause delay on the meeting proceedings. It is even more time consuming if the there are several file documents to be used in the meeting. Furthermore, these systems use streaming mechanism to deliver the slide presentation to each connected user. However, images loaded as slides becomes blurred, distorted, and have poor quality as compared to the original. This has been widely reported problem on Flash-based images, and 
hopefully solution will be on its way. On the brighter side, most web-based conferencing technologies today have built-in audio-video communication support.

\section{WebELS platform overview System design concepts}

WebELS is designed to provide an advanced e-Learning platform for globalizing higher education in science and technology focusing on authoring and dissemination of multimedia contents, aiming to assist instructors to archive their learning materials on the web for on-demand learning and to support online meeting [22].

The design concepts of WebELS can be summarized in the following:

- Supports asynchronous and synchronous e-Learning, i.e., on-demand self-learning and multi-location Internet-based meeting,

- Powerful and easy-to-use content authoring function that supports various documents,

- User-friendly interface for novice and non-IT users,

- Supports multi-operating systems and multi-browser,

- Must be available "anywhere and anytime" and must work even in strict firewall and proxy settings,

- Should be available not only in advanced countries where broadband Internet is widely used but also in developing countries such as in Asia and Africa where narrowband Internet is normally used [23].

\section{System components}

WebELS is a Java-based client-server system built with applets and JSP/servlets technology for client and server side programs, respectively. Java applets are used for content authoring and online slide presentation viewing, which requires the users' browser to have Java Runtime Environment (JRE) plug-in. For audio-video conference system, Red5 streaming server is used, and requires the users to have Adobe Flash Player plugin installed on their computer. The main components required for the WebELS server system are the following: Linux Operating System, Apache HTTP Server, Apache Tomcat, Open JDK, MySQL, Red5 Media Server, FFmpeg, and Apache OpenOffice.

\section{WebELS meeting module}

The overview of the WebELS Meeting system is shown in Figure 1. As shown, there are three servers in the server side system - database server, content server and streaming server. Database and content server are used for content and user data management, while streaming server is used for real-time audio-video communication.

Also in Figure 1, content authoring is done using the Java-based content Editor downloaded from the server. Content authoring using the Editor can achieve three operations, such as creating new content, editing content, and importing content from other servers. Existing file in various formats can be used, such as portable document format file (".pdf), slide presentation file (".ppt, ".pptx, ".odp), spreadsheet file (".xls, ". xlsx, ".ods) and word processing file (".doc, ".docx, ".odt). Files are converted to slide images either at the client or at the server. To create high-quality slides images, MS Office files are converted locally if there exists an MS Office application on the client system, 


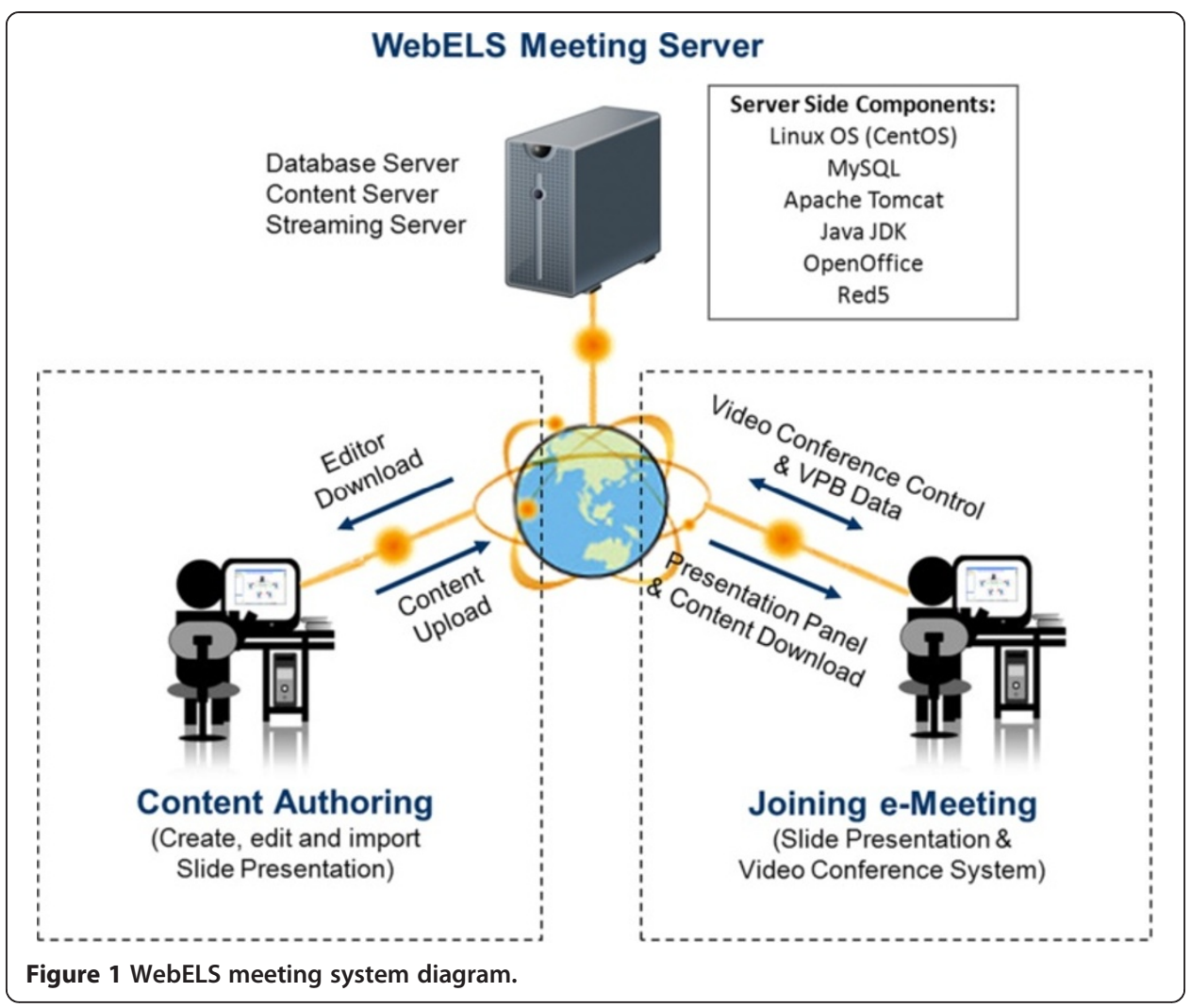

then it is uploaded to the server. When there is none, files are uploaded to the server and are automatically converted into images by using OpenOffice and GhostScript. PDF and OpenOffice files are converted at the server by default. Video files (".mpg, * mov, "avi) can also be embedded onto a special slide template which is possible to playback when viewing the content.

A virtual room is assigned to each created content and stored on the server. Each user accessing the same content will be able to join a meeting. In Figure 1, joining an e-Meeting requires the user to download the content and the presentation panel. The system stores the downloaded content onto the temporary folder, unzip the content package, and then load the slide images onto the presentation panel. The system prompts the user for username, and when done, displays the online presentation panel.

Online presentation has two user modes, namely (1) Presenter Mode, and (2) Listener Mode. In presenter mode, the user has over-all control of slide change, cursor position, zooming function and annotation function. Users in listener mode can only monitor who have joined the presentation, but does not have the rights to control the slides. The listener's presentation panel serves as a passive listener where it displays what is on the presenter's presentation panel.

The online presentation system implements the concept of a shared virtual presentation board. In this concept, a master copy of the presenter's presentation panel environment is stored onto the server, and used to achieve slide presentation synchronization between the presenter and the listeners. VPB data is updated by the presenter by sending the information in its presentation panel every time there is a user slide event, such 
as mouse click, mouse drag or button press. Listener of the same content retrieves the VPB data by polling the server, and thus be able to replicate in their presentation panel similar to what is in the presenter's presentation panel.

On the other hand, video conference system provides audio-video communication among the meeting participants who are in the same virtual room. Video conference and slide presentation panels are designed to be independent with each other, because one video conference panel can be used with multiple presentation content. Figure 2 shows the example of video conference and slide presentation.

\section{Synchronized online presentation}

\section{Online presentation panel}

WebELS Online Presentation Panel shown in Figure 3 is divided into four panels, namely (1) Presentation Display Panel, (2) Content Information Panel, (3) Control Panel, and (4) Collapsible Annotation Toolbar. Presentation display panel serves as the graphical screen of the slide presentation. Content information panel shows the content title and the slide navigator for quick slide changing. Control panel contains the slide control, zoom function, and presentation mode selection buttons. Annotation toolbar is hidden by default, but is displayed when annotation function is activated. It contains pen, pen color palette, pen size palette, eraser, and move tools.

\section{Online presentation features}

WebELS Online Presentation features the following important characteristics in implementing an effective e-Meeting system for higher-education:

- Slide Synchronization. A technique for real-time mirroring of slide presentation between the presenter and listener. The presentation control panel is equipped with slide control buttons (first slide, next slide, previous slide, and last slide) that

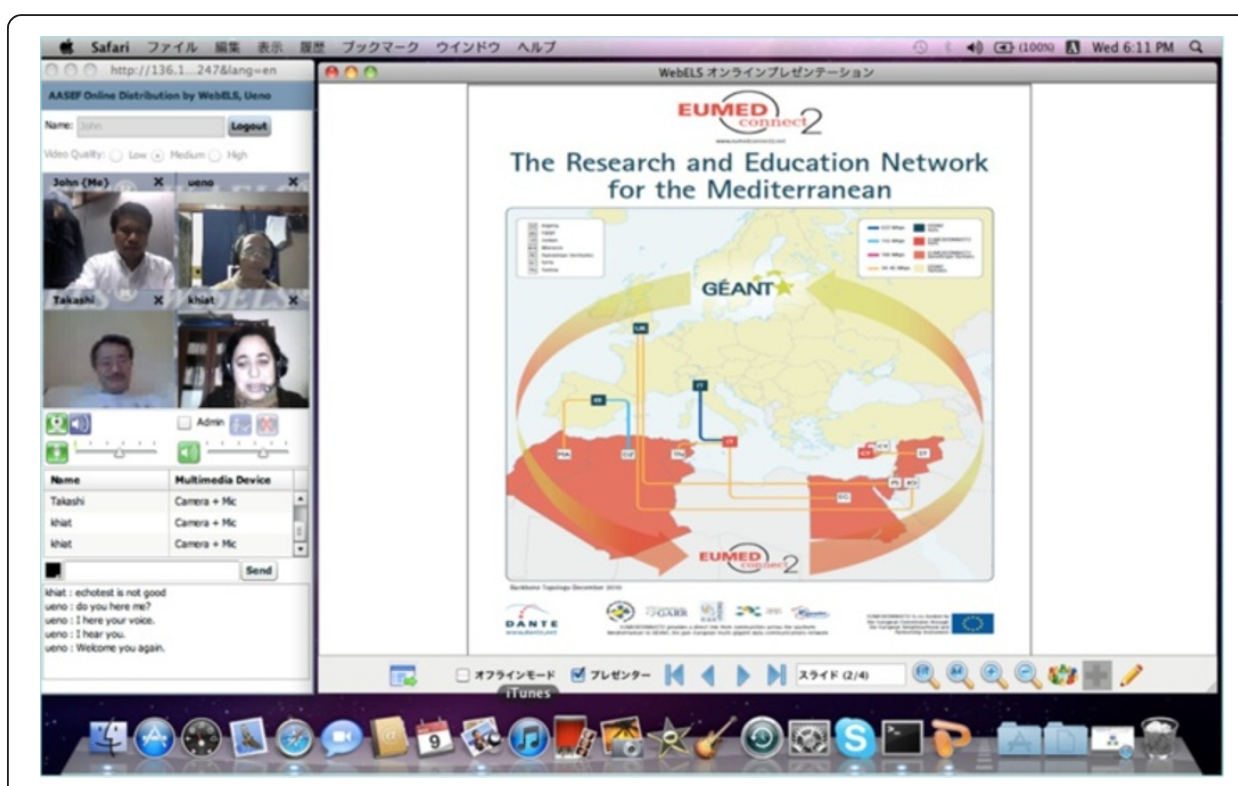

Figure 2 Online presentation and video conference (e-Meeting between Japan, U.K., and Algeria). 


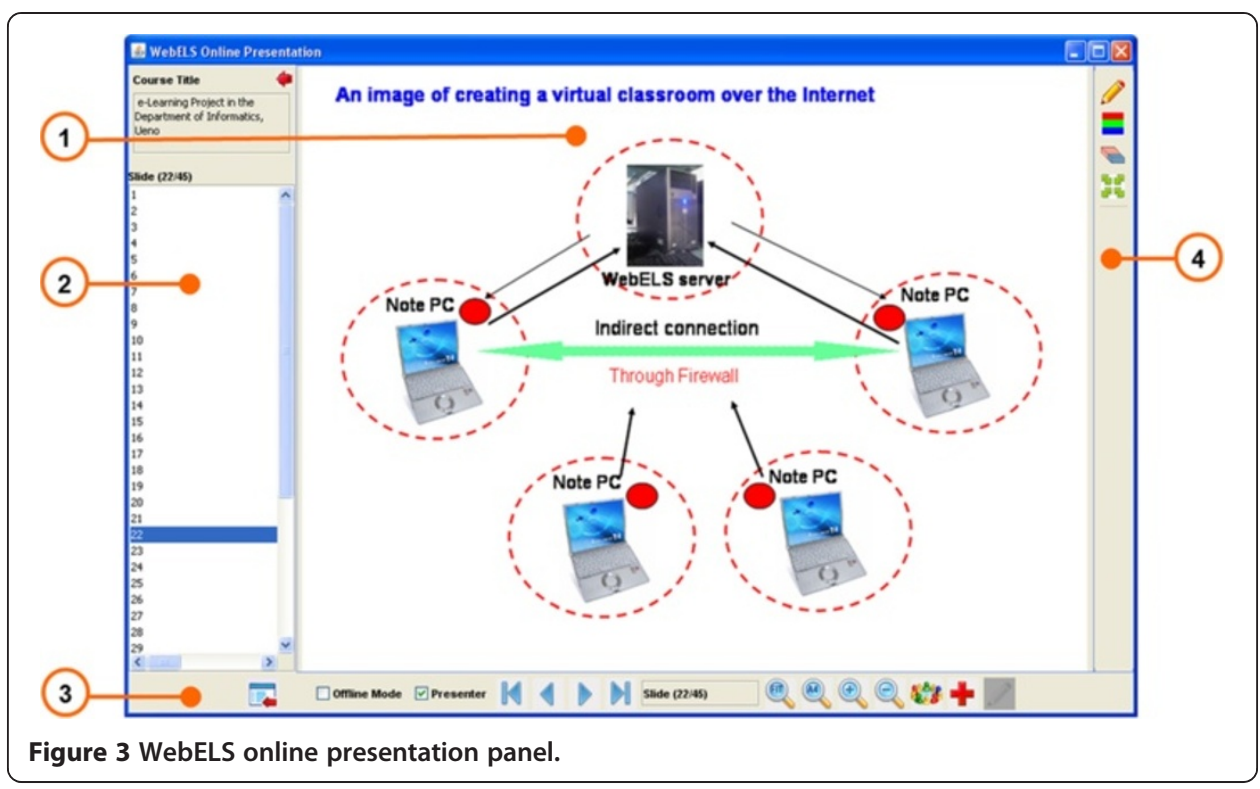

enables slide changing by the presenter, which is synchronized on the listeners' presentation display panel.

- Cursor-based Presentation. A heavy red crosshair cursor displayed as a pointer which guides the listener on which part of the slide is being presented. When the presenter moves the default cursor and clicks at a certain position on the presentation display panel, a heavy red crosshair cursor appears, and will also be displayed on the listeners' presentation display panel. See Figure 4a.

- Pen-based Presentation. A pen-like function during the presentation for writing annotation. Annotation on the presentation display panel is simply done like a freehand drawing. By pressing the left-hand mouse and holding it steadily, drag the thin crosshair cursor which in turn writes your desired object. Pen color and size can be selected. See Figure 4b.

- Slide Zoom Function. Slide zooming function is necessary when text or object on the slide are not readable or visible during online presentation as in Figure 4c. It is also worth mentioning that cursor and annotation function is also possible even in zoomed-in presentation display panel. The annotated object after zooming out is scaled down equally the same as the slide.

- Video Playback Function. Various video content formats (".mov, ".avi, ".mpg) can be embedded onto the slides. Video playback functions such as start, stop and pause are also made to synchronize between the presenter and the listener.

\section{Virtual presentation board concept}

The concept of a shared virtual presentation board is illustrated in Figure 5. The basic concept is to make a master copy of the presentation display panel of the presenter at the server, and it will be made available for the listeners to retrieve this data. The server keeps the online presentation panel applet, the presentation content, and the VPB data where it is created only when the content is used for the first time. Using a web browser, a client joins in a meeting by requesting to download the presentation panel and content in one compressed package. After downloading the package at the client 

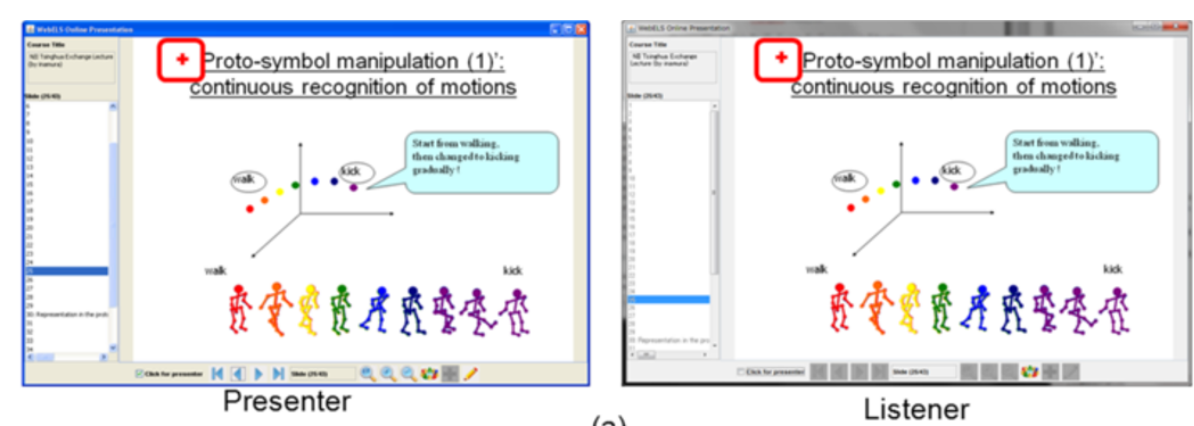

(a)

Listener
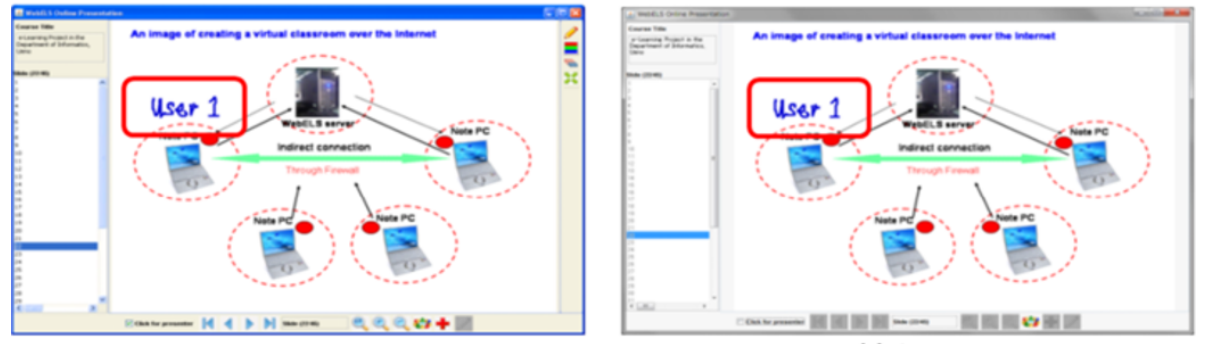

Listener

(b)

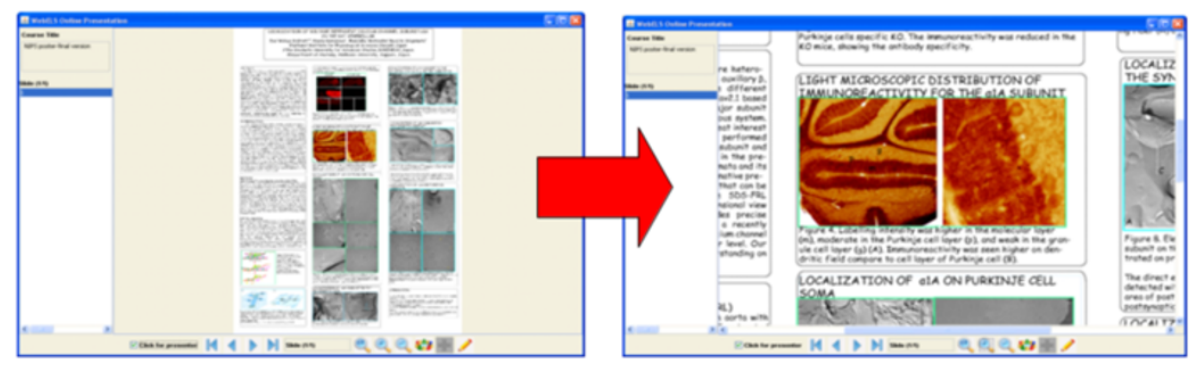

Fit Window

(c)

Zoom In

Figure 4 Examples of WebELS online presentation features. (a) Cursor synchronization function,

(b) Online annotation function, (c) Zooming function (Content courtesy of NIPS, Japan).

computer, it is uncompressed at the temporary folder, and the presentation panel loads the content accordingly. By default, the client is a listener which polls the server for VPB data. The retrieved data will be used to set the presentation display panel. When there is no existing presenter, the listener can become a presenter by selecting the option. To keep the meeting in order, only one client is allowed to become a presenter at a time, where switching of presenter's right is allowed. The presenter, having the right to control the presentation display panel, is the source of VPB data, wherein every slide event in its presentation display panel, a new VPB data is sent to the server. Slide events include slide changing, cursor positioning, slide zooming, scrollbar positioning, video playback, and annotation functions. At the server, a VPB data file is updated by a write method. For the listeners to mirror the presenter's display panel in a synchronized manner, the client system polls the server, reads and retrieves the VPB data if it exists, and refreshes the presentation display panel. Polling takes place every one second.

\section{VPB data structure}

Figure 6 shows the VPB data structure that consists of static and dynamic data. Static data structure is used for slide presentation objects that include presenter status, 


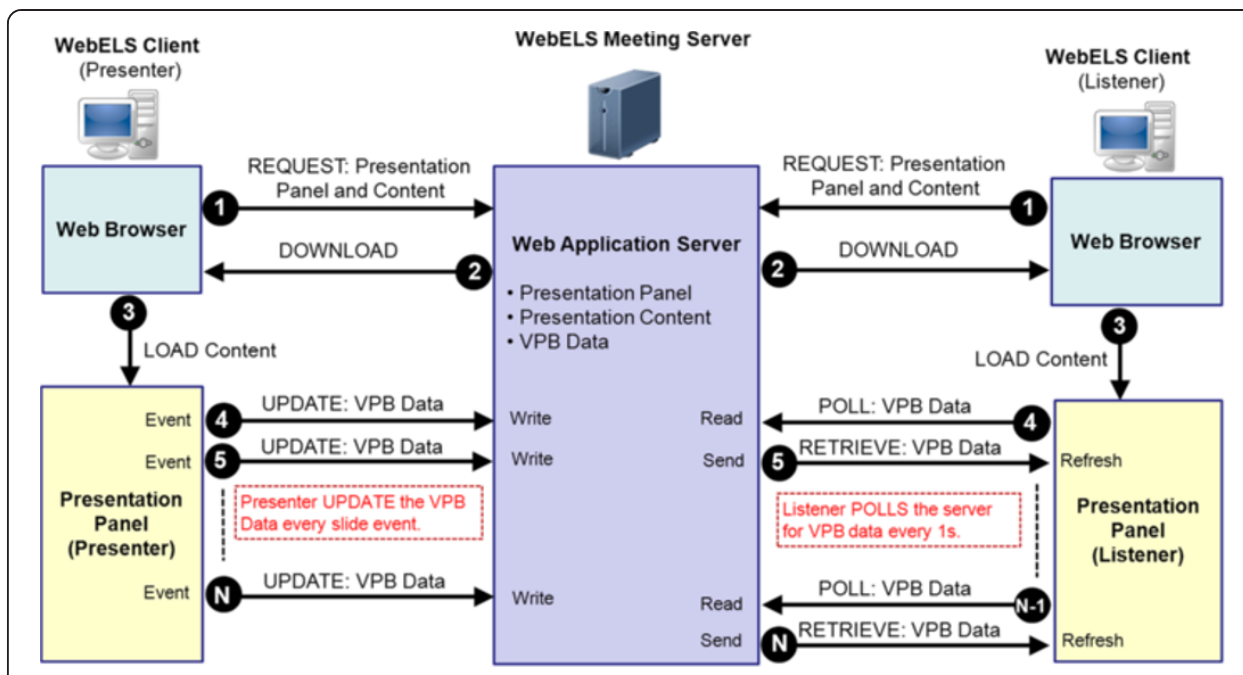

Figure 5 VPB concept operational flow.

presentation mode, slide number, cursor position, zoom data, scrollbar data and video function. These objects are used in cursor-based presentation. The part of the VPB data structure that has dynamic memory is used for the annotation data in a penbased presentation. It uses a vector type memory that is appropriate for growing array of objects.

The fields of the VPB static data structure are detailed in the following:

- presenter_status - an integer data type that tells whether there is a presenter in the online presentation or not. This flag is used to implement one-presenter policy.

- presentation_mode - an integer data type that tells the mode of presentation whether cursor-based or annotation-based.

- slide_number - an integer data type that tells the current slide being used by the presenter.

\begin{tabular}{|l|l|}
\hline \multicolumn{2}{|c|}{ VPB DATA STRUCTURE } \\
\hline \multicolumn{2}{|c|}{$\begin{array}{c}\text { Annotation Data } \\
\text { (Dtatic Data Structure) }\end{array}$} \\
\hline \\
\hline
\end{tabular}

Figure 6 VPB data structure. 
- cursor_xy - both an integer data type for the $\mathrm{x}$ value and $\mathrm{y}$ value of the cursor position relative to the display panel.

- current_zoom - both a double data type for current zoom-in scale and current zoom-out scale of the display panel.

- new_screen_zoom - both an integer data type that tells whether the display panel needs to be fixed or not after a zoom function.

- frame_size_xy - both an integer data type that tells the frame size of the presenter panel, which is used in calculating the listener's slide image to avoid mismatched display area.

- scrollbar_xy - both an integer data type for $\mathrm{x}$ and $\mathrm{y}$ scrollbar value that control horizontal and vertical view positions, respectively.

- video_mode - an integer data type that represents the mode for video functions whether start, pause, restart and stop.

- video_time - an integer data type that specifies the time in milliseconds when video mode is either stop or set time.

The VPB dynamic data are stored onto the vector memory that implements a growing array of objects. The dynamic data structure fields are detailed in the following:

- pen_color - three integer data types for RGB components of a color object.

- pen_size - float data type used for defining line width with default cap and joint styles rendered with Java Graphics2D object.

- start_mouseDragged_xy - both an integer data type for $\mathrm{x}$ and $\mathrm{y}$ values of cursor position relative to the display panel after the mouse is clicked, kept holding the left-button and dragged.

- end_mouseDragged_xy - both an integer data type for $\mathrm{x}$ and $\mathrm{y}$ values of cursor position relative to the display panel after the mouse is released from mouse dragged event.

\section{Audio-video communication support}

WebELS Meeting is equipped with a video conferencing system that provides audiovideo communication among the users in a shared virtual room. With this system, effective e-Meeting can take place because users can discuss in a face-to-face like environment alongside with the online synchronized slide presentation.

The video conference system adopted by WebELS uses Real Time Messaging Protocol (RTMP). RTMP is a protocol used for streaming audio, video and data over the Internet between Flash player on client side and streaming server. The Red5 streaming server uses port 443 (the default for HTTPS) as RTMP port, however it switches automatically to RTMP Tunneled (RTMPT) when the network communication cannot transverse through firewall due to security issues of certain network location. In RTMPT, RTMP data is encapsulated and exchanged via HTTP, and messages from the client, i.e., the Flash media player, are addressed to port 80 (the default for HTTP) on the server. The reason that we don't use RTMPT from the beginning of the network connection is because the speed of communication via RTMPT is slower than RTMP, where we want to keep the quality of audio-video communication as high as possible.

Parameters for audio-video quality have been optimized in order for the system to adapt the users' network environment without suffering from bad audio-video 
quality. These parameters include video resolution, video frame rate, video encoding quality, and audio sampling rate are used to provide three video quality settings such as low, medium and high, which can be selected in manual or automatic mode.

The system has administrator functions for keeping an orderly flow of the e-Meeting. Several administrator functions are mute user, mute all, set presenter, and block user. The system also has an automatic reconnection function that monitors the network connection status. When the network connection is lost, it waits for the new connection to establish, and login process automatically starts again by using the latest user environment and conference information [24].

\section{Performance evaluation and discussion}

Presentation image quality

In WebELS Meeting online presentation implemented in Java, slide presentation files are converted into series of slide images, archived on a compressed package and stored on the server. Before starting a meeting, content must be downloaded from the server onto the client system, uncompressed to the temporary folder, and loaded onto the presentation panel. During the meeting, slide control is implemented using shared VPB. Since slide images are now in client system, high-quality images can be displayed on the presentation panel as shown in Figure $7 \mathrm{~b}$ and look exactly the same as the original image shown in Figure 7a. Inset in Figure 7b shows the zoomin image bearing smaller text information, but still readable.

As for the Flash-based online presentation systems, slide presentation file is also converted into series of slide images. During the meeting, when the presenter share the slide image, the URL link for that image is shared unto the server via a message stream, which in turn is shared to the connected clients. Clients get the image from the server using the URL link. Image quality in Flash is blurry that small text cannot be recognized anymore as shown in the inset in Figure 7c. The image sharing process is handled by a shared object class which offer real-time data and object sharing between multiple Flash application clients or remote server [25].

In presentation using screen sharing technology, image quality becomes degraded because it requires compression algorithms in order to transmit huge data in real-time. Sophisticated compression algorithms produce encoded image size around 100 kbytes for $800 \times 600$ true color screen image (i.e., 1.44 Mbytes in size) [26]. With this data size and moderate frame rate, however, it is still required to use broadband Internet for better quality. Image rendering at the client side experience flickering resulting to distorted image when the presenter changes the slide or scrolls the window that takes several seconds to completely display the image as shown in Figure $7 \mathrm{~d}$.

Comparing all three technologies for image quality in online presentation, WebELS Meeting based on Java has the best image quality than in Flash-based online presentation and screen sharing technology. Flash-based images are blurry that small texts are difficult to recognized, while presentation based on the screen sharing have flickering effect resulting to distorted image when changing slide or scrolling window.

\section{Online presentation data size}

Online presentation is achieved by sharing data between the remote user and the server. Shown in Table 1, the data size and the transmit frequency between the client and the server for various online presentation technology. For WebELS cursor-based presentation 
The Research and Education Network for the Mediterranean

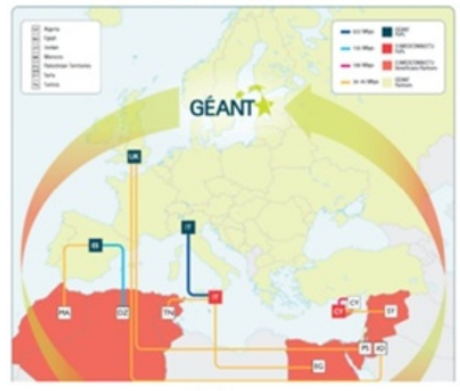

(a)

The Research and Education Network for the Mediterranean

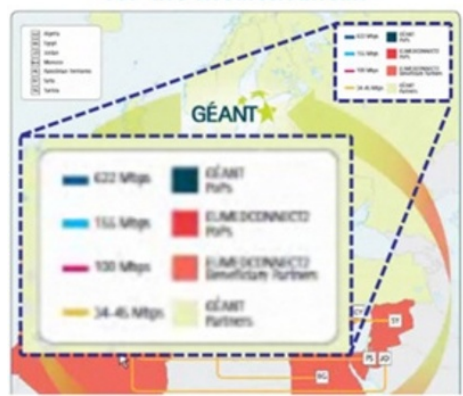

(c)

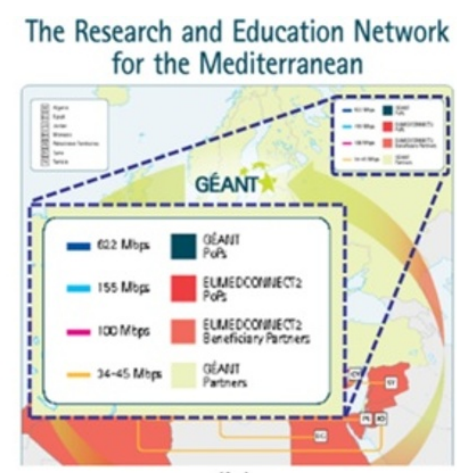

(b)

The Research and Education Network for the Mediterranean

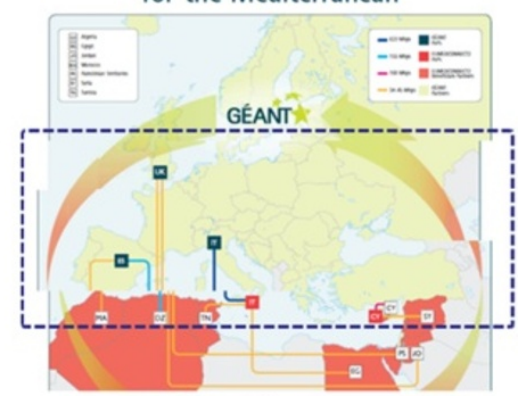

(d)

Figure 7 Slide presentation image comparison. (a) Original image, (b) WebELS slide presentation image with zoom-in text in the inset, (c) Flash-based slide presentation image with zoom-in text in the inset, (d) Screen sharing image with flickering.

mode, the VPB data contains slide control which range from 34 to 54 bytes, which is transmitted to the server for update every slide control events. For pen-based presentation mode, data size depends on how many the annotation is. In normal online meeting, annotation data range from 100 bytes to 10 kbytes, which is transmitted to the server for update every mouse drag release event. In Flash-based online presentation, sharing of image to other remote users is handled by a shared object class that sends the URL link of that image for other users to fetch. The typical image size of about 1 Mbytes is transmitted every slide change, while the annotation data also range from 100 bytes to 10 kbytes. For screen sharing technology, an $800 \times 600$ true color screen image after compression is about 100 kbytes. Screen image must be transmitted at a higher frame rate for better quality between the sender and receiver. In Table 1, the data size shared between the remote user and the server to achieve online presentation synchronization is comparatively minimal for WebELS than with the Flash-based online presentation and screen sharing technology.

\section{Client network bandwidth requirement}

Bandwidth utilization depends on the data size transmitted over a period of time between the client and the server. Based on the data in Table 1, WebELS VPB data for cursor-based presentation mode does not pose high bandwidth utilization since the data size is very small and slide control events are not that frequent. For VPB data in pen-based presentation mode, the more annotation is done, the larger the data sent 
Table 1 Online presentation data size and transmit frequency

\begin{tabular}{lll}
\hline Data types & Data size (bytes) & Transmit frequency \\
\hline WebELS VPB Data for cursor-based presentation mode & $34 \sim 54$ & Every slide control events \\
WebELS VPB Data for pen-based presentation mode & $100 \sim 10,000$ & Every mouse drag release \\
$\begin{array}{l}\text { Flash-based Slide Synchronization } \\
\text { (using SharedObject class for fetching the image) }\end{array}$ & $\approx 1,000,000$ & Every slide change \\
$\begin{array}{l}\text { Flash-based Slide Synchronization } \\
\text { (using SharedObject class for fetching annotation) }\end{array}$ & $100 \sim 10,000$ & Every mouse drag release \\
Compressed Screen Share Image Size & $\approx 100,000$ & Pre-set frame per second (fps) \\
\hline
\end{tabular}

every mouse release. As for the Flash-based online presentation, slide image is transferred from the server to the client every slide change. Though high quality images are typically around 1 Mbytes, slide change is not that frequent that would cause high bandwidth utilization. But in screen sharing technology, there is a requirement for broadband network to achieve better quality. Among the different online presentation technologies, VPB concept works well even in narrowband Internet environment, say a $56 \mathrm{kbps}$ line. On the other hand, slide synchronization delay between the presenter and listener using VPB is minimal because of its optimized data size, and delay would depend mainly on the network distance, i.e., round-trip propagation and transmission delay. Slide synchronization delay of 1 or 2 seconds is negligible in online distance presentation.

\section{Server requirements}

Synchronized online slide presentation consumes server resources for every connected user, but not as much as the video conferencing system do. For each video user connected to Red5 streaming server, CPU, memory, and I/O resources are utilized. However, with the current development in computer hardware technology, these are not very critical. The performance bottleneck that limits the number of users the server would accommodate with higher quality of service (QoS) depends on the network channel capacity.

The server uplink/downlink data rate with the number of users in a face-to-face meeting mode in an actual measurement is shown in Figure 8. Face-to-face meeting mode refers to a video conferencing scenario where all users use web-camera and microphone. Video qualities were optimized by setting the video parameters that results to average video stream bandwidth for low-quality, medium-quality and highquality as $72,120,240 \mathrm{kbps}$, respectively. Due to video-codec algorithm, video stream bandwidth fluctuates, i.e., gets higher when the image is in motion and gets lower when it is almost steady. The receive data rate at the server increases linearly with respect to the number of users, where each user uses the same video quality. As for the send data rate, it can be estimated by using the equation:

$$
B W_{\text {send }}=n *(n-1) * b w
$$

where: $B W_{\text {send }}$ - total send data rate at the server

$b w$ - bandwidth for one video stream

$n$ - number of users

In order to increase the number of concurrent users in face-to-face meeting mode, a conservative video quality should be used, i.e., low-quality having a video stream bandwidth of around $72 \mathrm{kbps}$. 


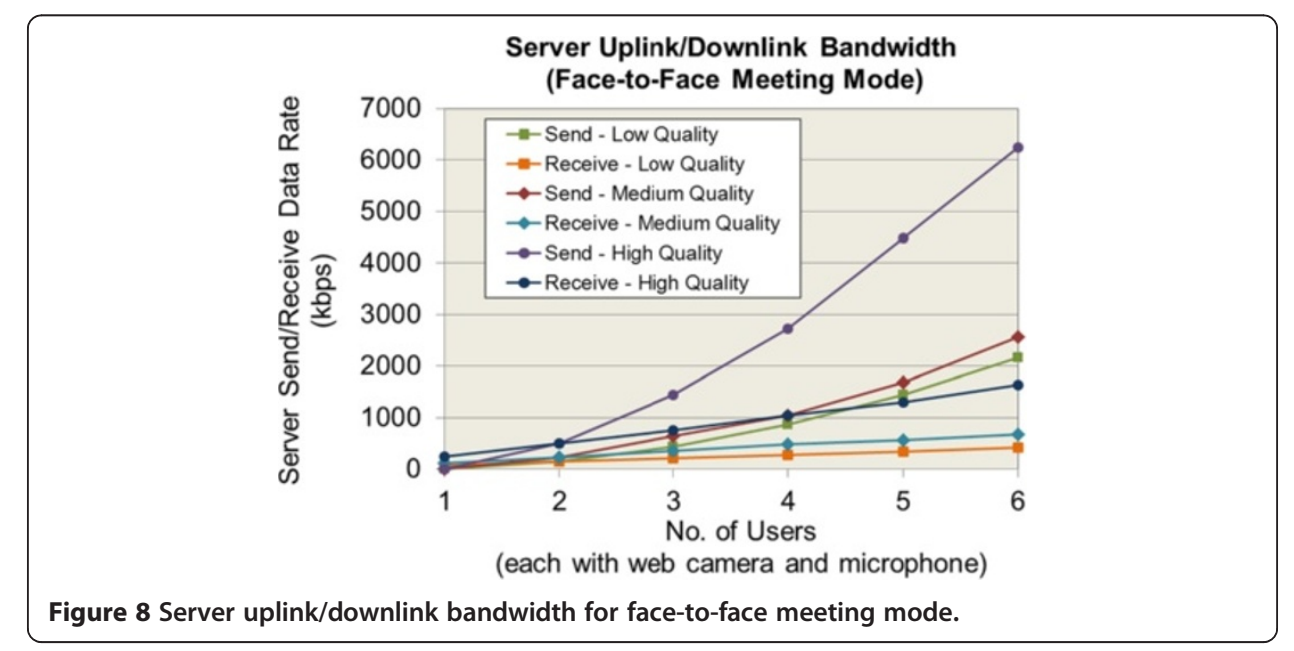

The server downlink data rate with the number of users in broadcast mode is shown in Figure 9. Broadcast mode is a usage scenario where the presenter use web camera to distribute video and share the slide presentation, but listeners do not use web camera. One example is distributing a presentation in a conference with remote viewers. The data in Figure 9 shows a linear increase in send data rate with the number of users. By selecting a conservative video quality for distribution, number of concurrent users can be increased largely in broadcast mode.

\section{Content-based meeting system}

WebELS Meeting is a content-based meeting system, i.e., content to be presented during the meeting must be pre-uploaded to the server before starting a meeting, although it is possible to upload another content when the meeting has already started. Since online presentation and video conference panels are separated, video conference connection can be kept while the meeting participants change their presentation panel to load another content. This scenario is efficient for a meeting with several participants where each has slide presentation to show, i.e., no wasted time for uploading new content

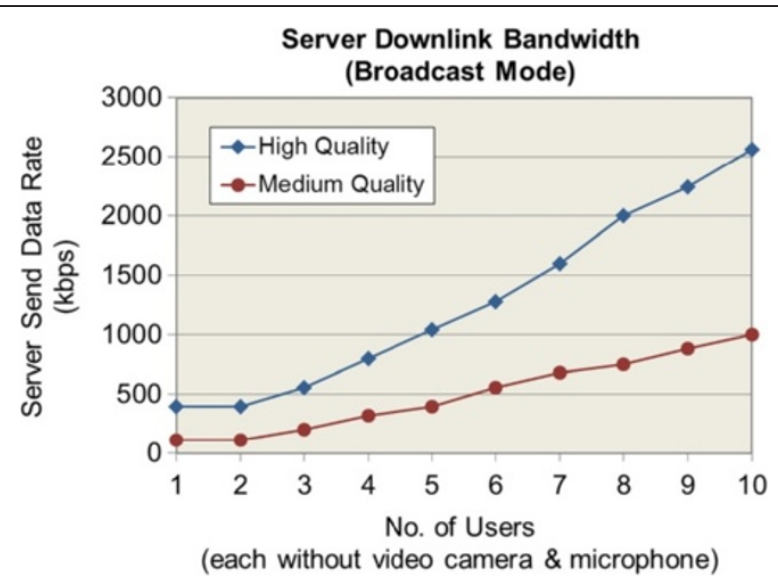

Figure 9 Server downlink bandwidth for broadcast mode. 
when changing the presenter. Unlike in Flash-based web-conferencing system that uploading of content is done on-the-fly. Uploading of content takes several seconds to few minutes for a large file since this process involves the conversion of uploaded file into series of slide images. Thus, content-based meeting system is also suitable for distributing live presentation of the conference proceedings to remote viewers because contents are already uploaded unto the server and changing of presentation does not consume much time. As an example, Figure 10 shows the list of presentation contents during the Asia-Arab Sustainable Energy Forum (AASEF) held at Nagoya, Japan last August 23-26, 2011.

\section{Summary}

This paper presented the online presentation based on a shared virtual presentation board (VPB) for e-Meeting on the WebELS platform. VPB is a data structure that defines several objects that represents the presentation panel of the presenter. In an e-Meeting, the presenter is the source of VPB data that is sent to the server, the server updates the master copy, the listener polls the server for the data periodically, and the retrieved data is used to set the presentation panel to achieve slide synchronization. Online presentation combined with video conference system creates a so-called virtual room for e-Meeting where participants convene via the Internet.

With VPB concept implemented on a content-based meeting system such as the WebELS Meeting, high-quality e-Meeting performance can be achieved. Meetings proceedings would not be interrupted by necessary on-the-fly uploading of content for presentation, since content must be pre-uploaded before a virtual meeting room is created. Because of this, the system is also suitable for distributing live presentation of the conference proceedings to remote viewers. Moreover, slide images are pre-downloaded on the users' local computer, thus there is no blurry and distorted slide presentation

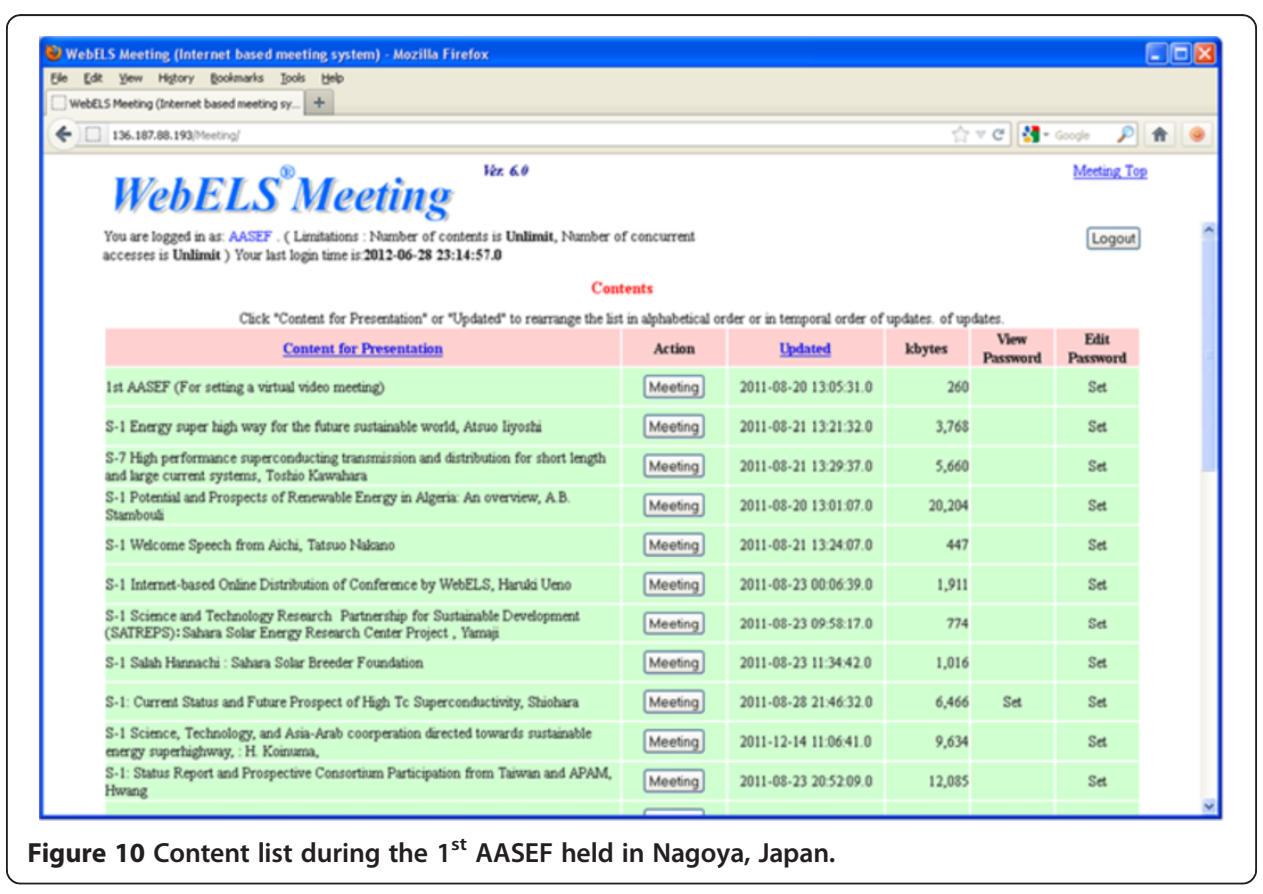


images. Online presentation can be attained smoothly because slide synchronization delay is minimized due to minimal VPB data that is shared between users and the server. More online presentation features are showcased, such as slide synchronization, cursor synchronization, freehand annotation function, zooming function, and embedded video playback. Moreover, the system works well in narrowband Internet as well as in a cloud environment.

\section{Competing interests}

The authors declare that they have no competing interests.

\section{Authors' contributions}

HU conceptualized the core functions of the Web-based E-Learning System (WebELS). AB carried out the design and implementation of shared virtual presentation board. All authors participated in the testing and evaluation of the system. $A B$ drafted the manuscript and did the final revision. All authors read and approved the final manuscript.

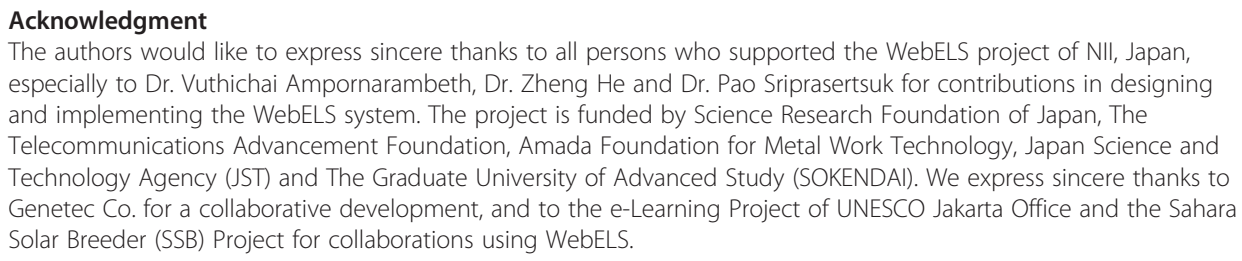
especially to Dr. Vuthichai Ampornarambeth, Dr. Zheng He and Dr. Pao Sriprasertsuk for contributions in designing and implementing the WebELS system. The project is funded by Science Research Foundation of Japan, The Telecommunications Advancement Foundation, Amada Foundation for Metal Work Technology, Japan Science and Technology Agency (JST) and The Graduate University of Advanced Study (SOKENDAI). We express sincere thanks to Genetec Co. for a collaborative development, and to the e-Learning Project of UNESCO Jakarta Office and the Sahara Solar Breeder (SSB) Project for collaborations using WebELS.

\section{Author details}

${ }^{1}$ National Institute of Informatics, Tokyo, Japan. ${ }^{2}$ The Graduate University for Advanced Studies, Tokyo, Japan.

Received: 11 January 2012 Accepted: 24 April 2013

Published: 26 April 2013

\section{References}

1. Ueno H (2002) Internet-Based Distance Learning for Lifelong Engineering Education - A Personal View and Issues. $J$ Inf Syst Educ 1(1):45-52

2. Blinco K, Mason J, McLean N, Wilson S (2004) Trends and Issues in E-Learning Infrastructure Development. A Whitepaper for alt-i-lab 2004. Prepared on behalf of DEST (Australia) and JISC-CETIS (UK). http://www.jisc.ac.uk/ uploaded_documents/Altilab04-infrastructureV2.pdf

3. Ampornarambeth V, Zhang T, Hadiana A, Shimamoto N, Ueno H (2006) A Web-Based e-Learning Platform for Postgraduate Education. Proceedings of the 5th IASTED International Conference on Web-based Education (WBE'06). ACTA Press, Anaheim, CA, USA, pp 388-393

4. Sriprasertsuk P, Berena AJ, Chunwijitra S, Ueno H (2009) A Study on an Open Source for Distance Real-Time Learning Environment. IEICE Technical Report, KBSE 2009-57:53-58

5. Ueno H, He Z, Yue J (2009) WebELS: A Content-Centered E-Learning Platform for Postgraduate Education in Engineering. Proceedings of the 13th International Conference on Human-Computer Interaction, Part IV: Interacting in Various Application Domains. Springer-Verlag Berlin Heidelberg, CA, USA, pp 246-255

6. Berena AJ, Sriprasertsuk P, He Z, Ueno H (2009) An Open Source Integrated e-Learning/e-Communication Platform for Postgraduate Education and Corporate Cyber Meeting. IEICE Technical Report, ET 109(193):33-37

7. (2011) Moodle. http://moodle.org

8. (2011) Joomla LMS. http://www.joomlalms.com

9. (2011) RealVNC. http://www.realvnc.com

10. (2011) Skype. http://skype.org

11. (2011) GoToMeeting. http://www.gotomeeting.com

12. (2011) WebEx. http://webex.com

13. (2012) TeamViewer. http://www.teamviewer.com

14. (2012) join.me. https://join.me

15. (2011) Google Docs. http://docs.google.com

16. (2011) SlideRocket. http://sliderocket.com

17. (2011) AuthorStream. http://www.authorstream.com

18. (2012) SlideShare. http://slideshare.com

19. (2012) V-Cube. http://vcube.com

20. (2012) Adobe Connect. http://adobe.com/products/adobeconnect.html

21. (2012) BigBlueButton. http://www.bigbluebutton.org

22. (2012) WebELS. http://webels.ex.nii.ac.jp

23. He Z, Yue J, Ueno H, WebELS (2009) A Multimedia E-Learning Platform for Non-broadband Users. Proceedings of the International Conference on Computer Engineering and Technology, pp 177-181 
24. Chunwijitra S, Berena AJ, Okada H, Ueno H (2011) Design of Suitable Meeting Management Model for WebELS Meeting to Meet the Business Situations. Proceedings of the First International Conference on Advanced Collaborative Networks, Systems and Applications, Luxembourg, pp 62-67

25. (2012) ActionScript Documentation Reference for Adobe Flash Platform. http://help.adobe.com/en_US/ FlashPlatform/beta/reference/actionscript/3/flash/net/SharedObject.html

26. Lin T, Hao P (2005) Compound Image Compression for Real-Time Computer Screen Image Transmission. IEEE Trans Image Process 14:993-1005

doi:10.1186/2192-1962-3-6

Cite this article as: Berena et al:: Shared virtual presentation board for e-Meeting in higher education on the

WebELS platform. Human-centric Computing and Information Sciences 2013 3:6.

Submit your manuscript to a SpringerOpen ${ }^{\circ}$ journal and benefit from:

- Convenient online submission

- Rigorous peer review

- Immediate publication on acceptance

- Open access: articles freely available online

- High visibility within the field

- Retaining the copyright to your article

Submit your next manuscript at $>$ springeropen.com 